\title{
Afecto positivo y negativo como mediador de la relación optimismo-salud: evaluación de un modelo estructural*
}

Positive and Negative Affect as Mediator of the Relatioship
Optimism-Health: Evaluation of a Structural Model

Recibido: febrero 10 de 2013 | Revisado: marzo 10 de 2014 | Aceptado: marzo 10 de 2014

\author{
Pablo Vera-Villarroel ** \\ Universidad de Santiago de Chile, USACH. \\ Karem Celis-ATENAS *** \\ Centro de innovación en tecnologías de la información \\ para aplicaciones sociales (CITIAPS) \\ Universidad de Santiago de Chile, USACH
}

doi:10.11144/Javeriana.UPSY13-3.apnm

Para citar este artículo: Vera-Villarroel, P. \& Celis-Atenas, K. (2014). Afecto positivo y negativo como mediador de la relación optimismo-salud: evaluación de un modelo estructural. Universitas Psychologica, 13(3), 1017-1026. http://dx.doi. org/10.11144/Javeriana.UPSY13-3.apnm

Esta investigación contó con el apoyo del proyecto FONDECYT № 1110520.

** Escuela de Psicología. Universidad de Santiago de Chile, USACH. Avenida Ecuador 3650, 3ํ Piso. Santiago. Correo electrónico: pablo.vera@usach.cl

***** Correo electrónico: pablo.vera@usach.cl

\section{RES U MEN}

Las variables psicológicas y especialmente la variables positivas han mostrado múltiples beneficios para los seres humanos. Importante evidencia indica que los sujetos con mejor bienestar, altos niveles de felicidad y emociones positivas muestran mejores indicadores de salud. El optimismo es una variable positiva ampliamente estudiada y con gran evidencia sobre sus beneficios en la salud. Sin embargo, modelos explicativos acerca de cómo se producen estos beneficios han sido menos estudiados. Se ha propuesto evaluar un modelo que establece cómo el optimismo puede ser una variable causal de la salud, mediada por los afectos positivos y negativos. Se realizó un análisis de modelo de ecuaciones estructurales para probar un modelo explicativo. Participaron 995 personas con edades entre los 18 y 60 años. Se evaluó el optimismo disposicional, afecto positivo y negativo, así como salud mental y física. Los resultados muestran que el ajuste al modelo es adecuado, permitiendo considerar al optimismo como una variable explicativa de la salud tanto física y como mental, mediado por afecto positivo y negativo. Palabras clave

optimismo; afecto positivo y negativo; salud física y mental

\section{A B S T R A C T}

Psychological variables and especially the positive variables have shown multiple benefits for humans. Important evidence indicates that subjects with better well-being higher levels of happiness and positive emotions show better health indicators. Optimism is a positive variable widely studied and strong evidence of its benefits on the health of individuals. However, an explanatory model about how these benefits occur has been less studied. It has been proposed to evaluate a model that establishes optimism can be a causal variable health mediated the positive and negative affects. An analysis of structural equation modeling was conducted to test an explanatory model. Participants 995 people aged between 18 and 60 years old. Dispositional optimism, positive and negative affect as well as mental and physical health was assessed. Structural model analysis was conducted to test an explanatory model. The results show that the model is suitable adjustment to consider allowing optimism as an explanatory variable of both physical and mental health mediated by positive and negative affect.

Keywords

optimism; positive and negative affect; physical and mental health 


\section{Introducción}

La relación entre variables psicologías y salud es ampliamente conocida y demostrada. Actualmente, se cuenta con evidencia en diferentes poblaciones, tanto angloparlantes como de población iberoamericana, entre las que se encuentra la expresión de rabia y enfermedades cardiacas (Davidson, Mostofsky \& Whang, 2010), la psiconeuroinmunología (Vera-Villarroel, 2001; Vera-Villarroel \& BuelaCasal, 1999), estilos de vida, conductas y salud (Camacho, Echeverría \& Reynoso, 2010; Gilts, Parker, Pettaway \& Cohen, 2013; Piqueras, Kuhne, Vera-Villarroel, van Straten \& Cuijpers, 2011; Vera-Villarroel, Piqueras, Kuhne, Cuijpers \& van Straten, 2014), conductas y VIH-SIDA (Piña, Sánchez-Sosa, Fierros, Ybarra \& Cázares, 2011; Ulibarri et al., 2010), cáncer (Arrivillaga, Correa, Varela, Holguín \& Tovar, 2006; Brown et al., 2013; Xu \& Roberts, 2010), entre otros.

En el último tiempo, diversos estudios han mostrado evidencia de la relación entre variables psicológicas positivas y salud (Contreras \& Esguerra, 2006; Csikszentimihalyí, 1997; Gable \& Haidt, 2005; Koopmans, Geleijnse, Zitman \& Giltay, 2010; Remor, Amorós \& Carboles, 2006; Seligman, Steen, Park \& Peterson, 2005), principalmente desde los trabajos de la psicología positiva (Park, Peterson \& Sun, 2013; Ruini \& Fava, 2013; Seligman, 2002; Seligman et al., 2005; Tarragona, 2013; Vázquez, 2013).

De todas las variables positivas estudiadas científicamente, es el optimismo la que en la actualidad cuenta con mayor evidencia empírica sobre sus beneficios en los seres humanos y específicamente en la salud. Los beneficios del optimismo han sido ampliamente documentados tanto en población clínica como no clínica y en diversos contextos, psicológico, de la salud, educativo, económico. Uno de los aspectos que más se ha verificado es que los sujetos optimistas presentan un mejor ajuste y afrontamiento a diversas situaciones estresantes, dado que sus esfuerzos los orientarían en obtener buenos resultados y mantendrían expectativas favorables de las situaciones (Chico, 2002; Londoño, 2009; López-Puga \& García, 2011; Peterson, 2000;
Xanthopoulou, Bakker \& Fischbach, 2013; Zenger et al., 2013).

De la misma forma, se ha documentado la relación entre optimismo y salud física (Carver, Sheier \& Segerstrom, 2010; Peterson, 2000; Peterson \& Bossio, 2001); optimismo y salud mental (Wilhelm, Wedguood, Parker, Geerligs \& Hadzi-Pavlovic, 2010; Wood \& Joseph, 2010); optimismo y mejor ajuste a las enfermedades y esperanza de vida (Hart, Vella \& Mohr, 2008; Taylor, Kemeny, Reed, Bower \& Gruenewald, 2000); optimismo y ansiedad, ira, depresión, stress (Abdel-Khalek, 2006; Brydon, Walker, Wawrzyniak, Chart \& Steptoc, 2009; Chico \& Ferrando, 2008; De Moor et al., 2006; Kivimäki et al., 2005; Lobel, DeVincent, Kaminer \& Meyer, 2000; Puskar, Ren, Bernardo, Haley \& Stark, 2008; Vera-Villarroel, Córdova-Rubio \& Celis-Atenas, 2009 a, 2009b; Vera-Villarroel, Pavez \& Mena, 2012; Vera-Villarroel et al., 2010); rasgos de personalidad y salud (Carver et al., 2010; Kam \& Meyer, 2012; Sharpe, Martin \& Roth, 2011).

Sin embargo, la mayoría de los estudios son habitualmente de tipo descriptivo o correlacional, sin contar en la actualidad con modelos explicativos consensuados o evidencia sustantiva relacionada al rol predisponente y/o causal del optimismo sobre estas variables y beneficios.

Específicamente relacionado con la salud se ha mostrado los beneficios del optimismo sobre la salud física y psicológica mostrando una relación positiva entre estos constructos, aun faltando evidencia de posibles modelos explicativos relacionados con estos hallazgos.

Debido a no contar en el último tiempo con algún modelo consensuado explicativo de estos beneficios se han propuesto en el último tiempo diversas aproximaciones que intentan dilucidar en términos causales los beneficios asociados al optimismo. Ejemplo de ello es lo propuesto por Lench (2010), el cual se basa en la teoría de la autorregulación (Carver \& Scheir, 1990), para establecer un posible mecanismo explicativo. Este autor describe que la relación entre optimismo y salud se daría considerando los objetivos diarios específicos y las emociones negativas; propone que estas serían mediadoras entre el optimismo y óptimos niveles 
de salud física y mental. Esta hipótesis la comprobó en un conjunto de estudiantes universitarios, encontrando como los objetivos de vida de los estudiantes median la relación entre optimismo y salud, siendo las metas de evitación las que mejor predicen la relación entre estas variables. No encontró, sin embargo, evidencia suficiente de que las expectativas positivas fuesen un buen mediador entre optimismo y salud. Este autor trabaja de forma indiferenciada los constructos de emociones y expectativas negativas (o positivas), por otra parte, pone de relieve que los objetivos orientados a metas con resultados negativos serían mejores predictores de la salud de las personas.

Otro ejemplo relacionado con modelos explicativos de los beneficios del optimismo y el rol de las emociones tanto positivas como negativas, es lo propuesto por Vera-Villarroel, Pavez y Silva (2012). Este modelo propone que el optimismo podría ser la variable psicológica aprendida desde la infancia, lo que lleva a experimentar mayor frecuencia de emociones positivas sobre las negativas a lo largo de la vida. Esta interacción es la que podría llevar a que los sujetos en los que se da esta condición presenten mejores niveles en los indicadores de bienestar, en conjunto con todos los beneficios ya verificados empíricamente en los diversos estudios disponibles.

De acuerdo a esto, el objetivo del presente estudio fue verificar parte del modelo de Vera-Villarroel et al. (2012) y explorar el rol causal del optimismo en la salud física y mental mediada por los afectos negativo y positivo (ver Figura 1).

La hipótesis del presente trabajo sostiene que el optimismo sería una variable explicativa de la salud (tanto física como psicológica), pero que esta relación estará mediada por los afectos. De la misma forma, esta mediación será diferente de acuerdo a la naturaleza de los afectos. Específicamente, se espera una mediación positiva con los afectos positivos y una mediación negativa con los afectos negativos (ver Figura 1 para observar el modelo que se debe verificar en el estudio).

\section{Método}

\section{Participantes}

En este estudio participaron 995 personas, de las cuales 433 eran hombres y 562 mujeres, entre 18 y 60 años de edad, con una media de 31.42 ( $\mathrm{DE}=$ 11.35); se consideró como criterio de exclusión que los participantes tuviesen diagnóstico de patología mental.

\section{Instrumentos}

Cuestionario de Orientación Vital Revisado ([LOTR]; Scheier, Carver \& Bridges, 1994, en la versión española de Otero-López, Luengo, Romero, Gómez $\&$ Castro, 1998). Cuestionario ampliamente utilizado para la evaluación del optimismo disposicional, compuesto por 10 ítems, donde seis evalúan optimismo y los restante cuatro son utilizados para no hacer tan evidente el contenido del cuestionario con una escala Likert de 5 puntos que va de 0 (totalmente en desacuerdo) a 4 (totalmente de acuerdo). La adaptación española obtiene una estimación de la confiabilidad de 0.7 y en la adaptación en muestra chilena fue de 0.65 (Chico, 2002; Ferrando, Chico \& Tous, 2002; Guillem-Riquielme \& Buela-Casal,

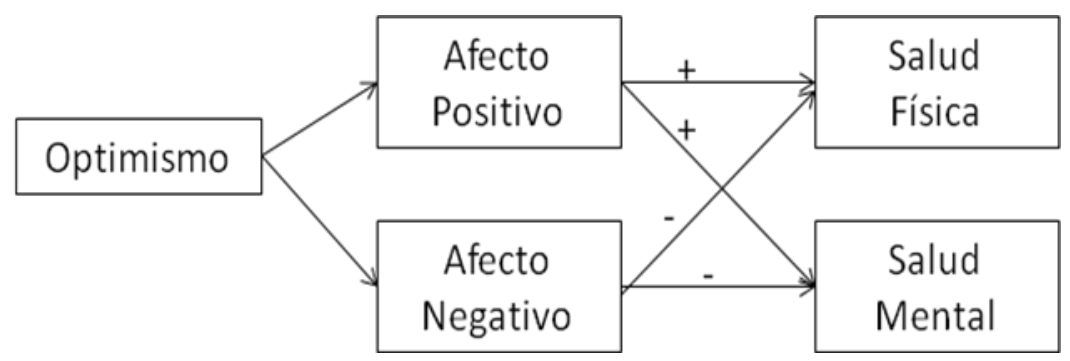

Figura 1: Salud física y mental será explicada por optimismo mediado por afecto positivo y negativo Fuente: elaboración propia 
2013; Vera-Villarroel, Córdoba-Rubio \& CelisAtenas, 2009a, 2009b).

Cuestionario de Afecto Positivo y Negativo ([PANAS]; Watson, Clark \& Tellegen, 1988 en la versión en español de Sandín et al., 1999). Cuestionario que a través de 20 preguntas evalúa afecto positivo y negativo en una escala Likert de 1 ( Nada o Casinada) a 5 (Muchísimo); cuenta con adecuados indicadores de confiabilidad y validez en muestras tanto de universitarios como en población general (Dufey \& Fernández, 2012; Sandín et al., 1999; Vera-Villarroel, Silva, Urzúa, Celis-Atenas \& Zych, en revisión; Watson et al., 1988).

Cuestionario SF-12 (Ware, Kosinski \& Keller, 1996) evalúa calidad de vida relacionada con la salud por medio de 12 ítems que miden estado positivo y negativo tanto de la salud física y mental de las personas. Las investigaciones que utilizan los doce ítems del SF, han verificado que este instrumento es una medida válida y confiable, encontrándose estimaciones de consistencia interna superiores a 0.7 y correlaciones significativas entre las versiones de la escala (Vera-Villarroel, Silva, Celis-Atenas \& Pavez, en Prensa).

TABLA 1

Media y desviación estándar para variables de estudio

\section{Procedimiento}

Personal capacitado realizó aplicación de conjunto de los cuestionarios utilizados para este estudio, quien previamente explicaba los alcances de la investigación y hacía entrega de consentimiento informado. Cada uno de los participantes respondió de forma voluntaria y sin pago de compensación por su participación.

\section{Análisis de datos}

Para el análisis de datos se utilizó programa SPSS versión 17 para análisis de estadísticos descriptivos y correlaciones entre las variables; para evaluación del modelo de ecuaciones estructurales se utilizó programa AMOS versión 17.

\section{Resultados}

A continuación en la Tabla 1, se presentan estadísticos descriptivos para las variables de estudio.

En la Tabla 2 se presentan correlaciones obtenidas entre las variables de estudio, en todos los casos

\begin{tabular}{lcc}
\hline & Media & DE \\
\hline Optimismo & 16.92 & 3.86 \\
Afecto Positivo & 34.78 & 7.79 \\
Afecto Negativo & 19.1 & 6.55 \\
Salud Física & 484.01 & 93.33 \\
Salud Mental & 334.06 & 55.19 \\
\hline
\end{tabular}

Fuente: elaboración propia

TABLA 2

Correlación de Pearson entre variables de estudio

\begin{tabular}{lcccc}
\hline & 1 & 2 & 3 & 4 \\
\hline Optimismo & & & & \\
Afecto Positivo & $0.51^{* *}$ & & & \\
Afecto Negativo & $-0.41^{* *}$ & $-0.3^{* *}$ & & \\
Salud Física & $0.28^{* *}$ & $0.34^{* *}$ & $-0.31^{* *}$ & $0.26^{* *}$ \\
Salud Mental & $0.26^{* *}$ & $0.34^{* *}$ & $-0.27^{* *}$ & \\
\hline
\end{tabular}

${ }^{*} p<0.05 . * * p<0.01$

Fuente: elaboración propia 
existen correlaciones significativas entre variables, donde optimismo correlaciona de forma positiva con afecto positivo, salud física y mental y de forma negativa con afecto negativo.

El modelo estructural fue evaluado con método de máxima verosimilitud (ver Figura 1). La adecuación del modelo se basa en prueba de chi-cuadrado, índice de ajuste comparativo (CFI) y la raíz del error medio cuadrático de aproximación (RMSEA) y correlación entre errores. En la Tabla 3 se presentan indicadores de ajuste de modelo evaluado.

En la Figura 2 se presenta modelo resultante con los pesos estandarizados obtenidos para cada una de las variables evaluadas. En resumen, la salud física y mental se puede explicar de forma adecuada por el optimismo mediado por afecto positivo y negativo.

\section{Discusión}

El presente estudio tuvo por objetivo evaluar modelo explicativo de la salud física y mental, a partir del optimismo y mediada esta relación por el afecto positivo y negativo. Los resultados obtenidos muestran buen ajuste del modelo.

Lo encontrado confirma que la variable optimismo podría actuar como variable causal de los beneficios en el bienestar, tradicionalmente encontrados en la literatura disponible. De la misma forma, los resultados sugieren que las habituales relaciones beneficiosas del bienestar y salud podrían estar causadas o influidas por otras variables, previamente relevantes en la vida de los seres humanos que no son tradicionalmente evaluadas en los estudios o al menos no valoradas como variables previas o causales a las condiciones de bienestar.

Estos resultados aportan evidencia al modelo teórico propuesto por Vera-Villarroel et al. (2012), donde se propone que el optimismo (entendido como un estilo aprendido de comportamiento) sería la condición previa para generar beneficios en el bienestar de las personas. El optimismo como un recurso que se aprende se ha sustentado tanto empírica como teóricamente. De la misma forma también se cuenta con evidencia de que esta condición se aprende desde la infancia (Avia \& Vázquez, 2013; Seligman, 2011a, 2011b). Por tanto, el contar con evidencia que el optimismo es una variable predisponente de la salud tanto física como mental se podría incluir como un factor relevante en la prevención y promoción de la salud y en el fomento de estilos de crianza o modelamiento, como estrategia de prevención de problemas psicopatológicos o, como sugiere el presente estudio, en mejores condiciones de salud en el futuro.

De la misma forma, la mediación de los afectos también ha sido verificada. Como se puede apre-

TABLA 3

Índices de ajuste obtenidos para modelo

\begin{tabular}{ccccccc}
\hline & $c^{2}$ & gl & $c^{2} / g l$ & CFI & RMSEA & I.C. RMSEA \\
\hline Modelo & 6.259 & 2 & 3.13 & 0.995 & 0.046 & $0.007-0.089$ \\
\hline
\end{tabular}

Fuente: elaboración propia

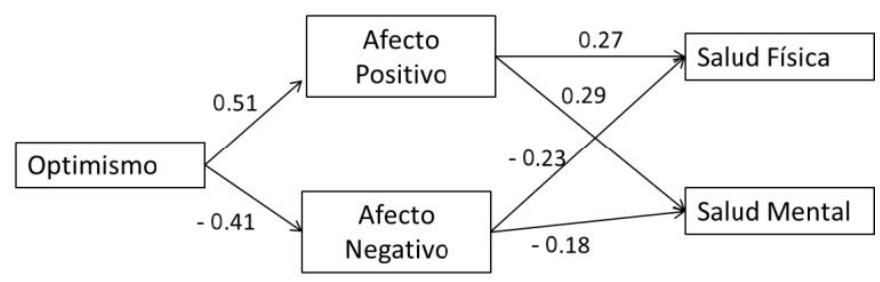

Figura 2: Modelo explicativo de salud física y mental a partir del optimismo, mediado por afecto positivo y negativo.

Fuente: elaboración propia

| Universitas Psychologica | V.13 | No.3 | Julio-septiembre | 2014 
ciar, los afectos median esta relación y tal como se esperaría previamente a nivel teórico, los resultados muestran que esta mediación está vinculada con la característica de los afectos. Así, los afectos positivos mediaron en términos positivos con mejores niveles de salud y, por otra parte, los afectos negativos mediaron en términos negativos. Resulta interesante, por lo tanto, verificar el efecto diferenciador en la salud dependiendo de la naturaleza de la afectividad.

El presente estudio muestra entonces evidencia que estas variables pueden estar explicando los beneficios del bienestar. Sin embargo, los resultados deben tomarse con cautela. Si bien se presenta evidencia de un modelo causal, esta evidencia es obtenida de indicadores tomados en un momento dado. Idealmente, la forma de obtener esta evidencia sería de estudios longitudinales con múltiples mediciones a lo largo de la vida, lo cual se sabe que es difícil de realizar por cuestiones prácticas. Sin embargo, mayor cantidad de investigaciones como el presente estudio son necesarias, y podrían entregar mayor evidencia sobre posibles variables causales del bienestar y sus beneficios de modo de mejorar las estrategias de prevención e intervención psicológicas y de las ciencias de la salud.

\section{Referencias}

Abdel-Khalek, A. (2006). Measuring happiness with a single-item scale. Social Behavior and Personality, 34(2), 139-150.

Arrivillaga, M., Correa, D., Varela, M., Holguín, L., \& Tovar, J. (2006). Variables psicológicas en mujeres diagnosticadas con VIH/SIDA: un estudio correlacional. Universitas Psychologica, 5(3), 659-668.

Avia, D., \& Vázquez, C. (2013). Optimismo inteligente. Madrid: Alianza Editorial.

Brown, L., Rand, K., Bigatti, S., Stewart, J., Theobald, D., Wu, J., \& Kroenke, K. (2013). Longitudinal relationship between fatigue and depression in cancer patients with depression and/or pain. Health Psychology, 32(12), 1199-1208. http://dx.doi. org/10.1037/a0029773

Brydon, L., Walker, C., Wawrzyniak, A. J., Chart, H., \& Syeptoc, A. (2009). Dispositional optimism and stress-induced changes in immunity and negative mood. Behavioral and Inmunity, 23(6), 810-816.

Camacho, L., Echeverría, S., \& Reynoso, L. (2010). Estilos de vida y riesgos en la salud de trabajadores universitarios. Journal of Behavior, Health Eु Social Issues, 2(1), 91-103. http://dx.doi.org/10.5460/jbhsi. v2.i1.10

Carver, C. S., \& Scheier, M. (1990). Principles of selfregulation: Action and emotion. En T. E. Higgins \& R. M. Sorrentino (Eds.), Handbook of motivation and cognition: Foundations of social behavior (Vol. 2, pp. 3-52). New York: Guilford Press.

Carver, C. S., Scheier, M. F., \& Segerstrom, S. C. (2010). Optimism. Clinical Psychology Review, 30 (7), 879. 889. http:/dx.doi.org/10.1016/j.cpr.2010.01.006

Contreras, F., \& Esguerra, G. (2006). Psicología positiva: una nueva perspectiva en Psicología. Diversitas. Perspectivas en Psicología, 2(2), 311-319.

Chico, E. (2002). Optimismo disposicional como predictor de estrategias de afrontamiento. Psicothema, 14(3), 544- 550.

Chico, E., \& Ferrando, P. (2008). Variables cognitivas y afectivas como predictoras de satisfacción en la vida. Psicothema, 20(3), 408-412.

Csikszentimihalyí, M. (1997). Finding flow. New York: Basic Books.

Davidson, K. W., Mostofsky, E., \& Whang, W. (2010). Don't worry, be happy: Positive affect and reduced 10-year incident coronary heart disease: The Canadian Nova Scotia Health Survey. European Heart Journal, 31(9), 1065-1070.

De Moor, J. S., De Moor, C. A., Basen-Engquist, K., Kudelka, A., Bevers, M. W. \& Cohen, L. (2006). Optimism, distress, health-related quality of life, and change in cancer antigen 125 among patients with ovarian cancer undergoing chemotherapy. Psychosomatic Medicine, 68(4), 555-562.

Dufey, M., \& Fernández, A. M. (2012). Validez y confiabilidad del Positive Affect and Negative Affect Schedule (PANAS) en estudiantes universitarios chilenos. Revista Iberoamericana de Diagnóstico y Evaluación Psicológica, 34(2), 157-173.

Ferrando, P., Chico, E., \& Tous, J. (2002). Propiedades psicométricas del test de optimismo Life Orientation Test. Psicothema, 14(3), 673-680. 
Gable, S., \& Haidt, J. (2005). What (and why) is positive psychology? Review of General Psychology, 9(2), 103-110.

Gilts, C., Parker, P., Pettaway, C., \& Cohen, L. (2013). Psychosocial moderators of presurgical stress management for men undergoing radical prostatectomy. Health Psychology, 32(12), 1218-1226. http:// dx.doi.org/10.1037/a0030189

Guillén-Riquelme, A., \& Buela-Casal, G. (2013). Versión Breve del STAI en adolescentes y universitarios españoles. Terapia Psicológica, 31(3), 293-299.

Hart, S., Vella, L., \& Mohr, D. (2008). Relationships among depressive symptoms, benefit-finding, optimism, and positive affect in multiple sclerosis patients after psychotherapy for depression. Health Psychology, 27(2), 230-238.

Kam, C., \& Meyer, J. P. (2012). Do optimism and pessimism have different relationships with personality dimensions? A re-examinatio. Personality and Individual Differences, 52(2), 123-127. http://dx.doi. org/10.1016/j.paid.2011.09.011

Kivimäki, M., Elovainio, M., Singh-Manoux, A., Vahtera, J., Helenius, H., \& Pentti, J. (2005). Optimism and pessimism as predictors of change in health after death or onset of severe illness in family. Health Psychology, 24(4), 413-421.

Koopmans, T. A., Geleijnse, J. M., Zitman, F. G., \& Giltay, E. J. (2010). Effects of happiness on allcause mortality during 15 years of follow-up: The Arnhem elderly study. Journal of Happiness Studies, $11,113-124$.

Lench, H. (2011). Personality and health outcomes: Making positive expectations a reality. Journal of Happiness Studies, 12(3), 493-507. http://dx.doi. org/10.1007/s10902-010-9212-z

Lobel, M., DeVincent, C., Kaminer, A., \& Meyer, B. (2000). The impact of prenatal maternal stress and optimistic disposition on birth outcomes in medically high-risk women. Health Psychology, 19(6), 544-553.

Londoño, C. (2009). Optimismo y salud positiva como predictores de la adaptación a la vida universitaria. Acta Colombiana de Psicología, 12(1), 95-107.

López-Puga, J., \& García, J. (2011). Optimismo, pesimismo y realismo disposicional en emprendedores potenciales de base tecnológica. Psicothema, 23(4), 611-616.

Otero-López, J. M., Luengo, A., Romero, E., Gómez, J. A., \& Castro, C. (1998). Psicología de la personalidad. Manual de prácticas. Barcelona: Ariel Practicum.

Park, N., Peterson, C., \& Sun, J. K. (2013). Positive psychology: Research and practice. Terapia Psicológica, $31(1), 11-20$.

Peterson, C. (2000). The future of optimism. American Psychologist, 55(1), 44-55.

Peterson, C., \& Bossio, L. (2001). Optimism and physical well-being. En E. Chang, (Ed), Optimism Eु pessimism: Implications for theory, research, and practice (pp. 127-145). Washington, DC: American Psychological Association. http://dx.doi. org/10.1037/10385-006

Piña, J. A., Sánchez-Sosa, J. J., Fierros, L. E., Ybarra, J. L., \& Cázares, O. (2011). Variables psicológicas y adhesión en personas con VIH: evaluación en función del tiempo de infección. Terapia Psicológica, 29(2), 149-157.

Piqueras, J. A., Kuhne, W., Vera-Villarroel, P., van Straten, A., \& Cuijpers, P. (2011). Happiness and health behaviours in Chilean college students: A cross-sectional survey. BMC Public Health, 11, 443 453. http://dx.doi.org/10.1186/1471-2458-11-443

Puskar, K., Ren, D., Bernardo, L. M., Haley, T., \& Stark, K. H. (2008). Anger correlated with psychosocial variables in rural youth. Issues in Comprehensive Pediatric Nursing, 31(2), 71-87.

Remor, E., Amorós, M., \& Carboles, J. (2006). El optimismo y la experiencia de ira en relación con el malestar físico. Anales de Psicología, 22(1), 37-44.

Ruini, C., \& Fava, G. A. (2013). The polarities of psychological well-being and their response to treatment. Terapia Psicológica, 31(1), 49-57.

Sandín, B., Chorot, P., Lostao, L., Joiner, T. E., Snated, M. A., \& Valiente, R. M. (1999). Escala PANAS de afecto positivo y negativo: validación factorial y convergencia transcultural. Psicothema, 11(1), $37-51$.

Scheier, M. F., Carver. C., \& Bridges, M. W. (1994). Distinguishing optimism from neuroticism (and trait anxiety, self-mastery, and self-esteem): A re- 
evaluation of the Life Orientation Test. Journal of Personality and Social Psychology, 67(6), 1063-1078. Sharpe, P. J., Martin, N. R., \& Roth, K. A. (2011). Optimism and Big Five factors of personality: Beyond neuroticism and extraversion. Personality and Individual Differences, 51(8), 946-951.

Seligman, M. E. P. (2002). Authentic happiness: Using the new positive psychology to realize your potential for lasting fulfillment. New York: Free Press/Simon and Schuster.

Seligman, M. E. P. (2011a). Aprenda optimismo. Barcelona: Random House Mondadori S.A.

Seligman, M. E. P. (2011b). La vida que florece. Barcelona: Ediciones B.

Seligman, M. E. P., Steen, T., Park, N., \& Peterson, C. (2005). Positive psychology progress. American Psychologist, 60(5), 410-421. http://dx.doi. org/10.1037/0003-066x.60.5.410

Tarragona, M. (2013). Positive psychology and constructive therapies: An integrative proposal. Terapia Psicológica, 31(1), 115-126.

Taylor, S., Kemeny, M., Reed, G., Bower, J., \& Gruenewald, T. (2000). Psychological resources, positive illusions, and health. American Psychologist, 55(1), 99-109.

Ulibarri, M., Strathdee, S., Lozada, R., Magis-Rodriguez, C., Amaro, H., O’Campo, P., \& Patterson, T. (2010). Intimate partner violence among female sex workers in two Mexico-U.S. border cities: Partner characteristics and HIV risk behaviors as correlates of abuse. Psychological Trauma: Theory, Research, Practice, and Policy, 2(4), 318-325.

Vázquez, C. (2013). Psicología positiva: introducción al número especial (en memoria de Chris Peterson). Terapia Psicológica, 31(1), 5-9.

Vera-Villarroel, P. (2001). Aprendizaje de respuestas inmunológicas: evidencia experimental. Revista Colombiana de Psicología, 10, 21-28.

Vera-Villarroel, P., \& BueIa-Casal, G. (1999). Psiconeuroinmunología: relaciones entre factores psicológicos e inmunitarios en humanos. Revista Latinoamericana de Psicología, 31(2), 271-289.

Vera-Villarroel, P., Buela-Casal, G., Zych, I., Córdova-Rubio, N., Celis-Atenas, K., Zepeda, L., \& Spielberger, C. (2010). Chilean experimental version of the State-Trait Depression Questionnaire
(ST-Dep): State subscale (S-Dep). Psychological Reports, 106(1), 65-73. http://dx.doi.org/10.2466/ pr0.106.1.65-77

Vera-Villarroel, P., Córdova-Rubio, N., \& Celis-Atenas, K. (2009a) Optimismo versus autoestima: implicancia para la psicología clínica y la psicoterapia. Revista Argentina de Clínica Psicológica, 18(1), 21 30.

Vera-Villarroel, P., Córdova-Rubio, N., \& Celis-Atenas, K. (2009b). Evaluación del optimismo: un análisis preliminar del Life Orientation Test versión revisada (LOT-R) en población chilena. Universitas Psychologica, 8(1), 61-68.

Vera-Villarroel, P., Pavez, P., \& Mena, L. (2012). El rol de la felicidad y el optimismo como factor protector de la ansiedad. Universitas Psychologica, 11(2), 369-380.

Vera-Villarroel, P., Pavez, P., \& Silva, J. (2012). El rol predisponente del optimismo: hacia un modelo etiológico del bienestar. Terapia Psicológica, 30(2), 77-84.

Vera-Villarroel, P., Piqueras, J. A., Kuhne, W., Cuijpers, P., \& van Straten, A. (2014). Differences between men and women in self-reported body mass index and its relation to drug use. Substance Abuse Treatment, Prevention, and Policy, 9(1), 1-11. http://dx.doi. org/10.1186/10.1186/1747-597X-9-1

Vera-Villarroel, P., Silva, J., Celis-Atenas, K., \& Pavez, P. (en prensa). Evaluación del Cuestionario SF-12: verificación de la utilidad de la Escala Salud Mental. Manuscrito presentado para su publicación.

Vera-Villarroel, P., Silva, J., Urzúa, A., Celis-Atenas, K., \& Zych, I. (en revisión). Reliability and validity of the Chilean Positive and Negative Affect Schedule (PANAS). Manuscrito presentado para su publicación.

Ware, J. E., Kosinski M., \& Keller, S. D. (1996). A 12-item short form health survey. Medical Care, 34(3), 220-223.

Watson, D., Clark, L. A., \& Tellegen, A. (1988). Development and validation of brief measures of positive and negative affect: The PANAS scales. Journal of Personality and Social Psychology, 54(6), 1063-1070.

Wilhelm, K., Wedguood, L., Parker, G., Geerligs, L., \& Hadzi-Pavlovic, D. (2010). Predictingmental 
health and well-being in adulthood. Journal of Nervous and Mental Disorders, 198(2), 85-90.

Wood, A., \& Joseph, S. (2010). The absence of positive psychological (eudemonic) well-being as a risk factor for depression: A ten year cohort study. Journal of Affective Disorders, 122(3), 213-217.

Xanthopoulou, D., Bakker, A., \& Fischbach, A. (2013). Work engagement among employees facing emotional demands. The role of personal resources. Journal of Personnel Psychology, 12(2), 74-84. http:// dx.doi.org/10.1027/1866-5888/a000085
Xu, J., \& Roberts, R. (2010). The power of positive emotions: It's a matter of life or death-subjective well-being and longevity over 28 years in a general population. Health Psychology, 29(1), 9-19.

Zenger, M., Finck, C., Zanon, C., Jimenez, W., Singer, S., \& Hinz, A. (2013). Evaluation of the Latin American version of the Life Orientation TestRevised. International Journal of Clinical and Health Psychology, 13(1), 243-252. 
